RESEARCH ETHICS

\title{
A pilot study of the quality of informed consent materials for Aboriginal participants in clinical trials
}

\author{
F M Russell, J R Carapetis, H Liddle, T Edwards, T A Ruff, J Devitt
}

J Med Ethics 2005;31:490-494. doi: 10.1136/jme.2002.002279

\begin{abstract}
See end of article for authors' affiliations

.....................

Correspondence to: Dr J Carapetis, Centre for International Child Health, University of Melbourne Department of Paediatrics, Flemington Road, Parkville, Melbourne 3052, Australia; jonathan.carapetis@ rch.org.au

Received 20 October 2002 In revised form 15 January 2004 Accepted for publication 9 February 2004
\end{abstract}

\begin{abstract}
Objective: To pilot informed consent materials developed for Aboriginal parents in a vaccine trial, and evaluate their design and the informed consent process.

Methods: Cross sectional quantitative and qualitative survey of 20 Aboriginal and 20 non-Aboriginal women in Alice Springs. Information about the proposed research was presented to Aboriginal participants by an Aboriginal researcher, using purpose designed verbal, visual, and written materials. Non-Aboriginal participants received standard materials developed by the sponsor. Questionnaires were used to evaluate recall and understanding immediately and five days later. Qualitative analysis of Aboriginal participants' interviews was performed.

Results: There were no differences between the groups in understanding of diseases prevented by the vaccine, the potential risks of participating, or the voluntary nature of participation. Most Aboriginal participants had difficulty with the concept of a "licensed" versus "unlicensed" vaccine. The nonAboriginal group had a good understanding of this. Aboriginal participants identified the use of the flipchart, along with a presentation by a doctor and Aboriginal health worker, as preferred delivery modes. Group presentations were preferred rather than one-on-one discussions. The use of the questionnaire posed considerable methodological difficulties.

Conclusions: A one-off oral presentation to Aboriginal participants is unlikely to produce "informed consent". Key but unfamiliar concepts require identification and particularly considered presentation.
\end{abstract}

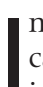
formed consent is a prerequisite for performing biomedical research. The ethical and legal requirements of consent include the provision of information and the capacity to make a voluntary choice. ${ }^{1}$ Ethical guidelines on conducting research in Aboriginal and Torres Strait Islander people address issues relating to important values to be considered, the relationship between researchers and communities, ownership and publication of data, consultation, and community involvement, including the need to gain informed consent from Aboriginal communities or their agencies before gaining individual informed consent. $^{23}$ However, there are no clear guidelines as to how consent should be obtained.

Resources are available to guide the development and design of effective printed health education materials for the Australian English speaking population,,$^{4-6}$ including a simple method to screen out poor materials. ${ }^{7}$ Information is often conveyed to Aboriginal people using videos, written information including flipcharts and participant information booklets, and verbally. Sometimes, these materials are translated into appropriate languages. However, the content, design, and delivery of informed consent materials for Aboriginal people have not been evaluated. Moreover, to our knowledge there are no previous reports evaluating whether Aboriginal people participating in research are informed to the extent that is ethically and legally required.

A study to determine the feasibility of conducting a clinical trial of an unlicensed conjugate pneumococcal vaccine (SmithKline Beecham; GlaxoSmithKline after 2001) was undertaken in the Northern Territory during 2000. The aim of the proposed trial was to determine the vaccine's effectiveness in reducing pneumonia and otitis media in Aboriginal children. The successful conclusion of the feasibility study was followed by an implementation phase of the vaccine trial itself. Although the trial was abandoned before enrolment of the first participant, informed consent materials had been developed.

We undertook a pilot project to evaluate the usefulness of these informed consent materials in conveying information to potential Aboriginal participants, in order to inform future studies in this population. In particular, we aimed to evaluate whether delivery of the materials would convey to Aboriginal people the difference between a licensed and unlicensed vaccine, the risks and benefits of participating in the vaccine trial, and the voluntary nature of participation.

\section{METHODS}

\section{Participants and setting}

The study was conducted in Alice Springs in February 2001. Because the vaccine trial was abandoned before the materials were translated into Aboriginal languages, we piloted the materials in English. Therefore, we planned to present them to Aboriginal mothers who spoke English as a first language. Twenty Aboriginal participants were recruited through networks of the Aboriginal members of the project team. This group represented a broad cross section of women from nearby small communities and urban Alice Springs. Due to last minute recruitment changes, this group of 20 ultimately included nine participants who spoke English as a second language. This unpredicted development led to a change in methodology (see below).

In order to compare the effectiveness of a purpose designed informed consent package for Aboriginal participants with the standard approach to informed consent in vaccine trials in non-indigenous populations, we included a comparison group of 20 non-Aboriginal mothers who were recruited through Alice Springs Hospital. These women received the same information as the Aboriginal participants, but prepared in a format standard for clinical trials in nonindigenous populations. 


\section{Informed consent material design}

The informed consent materials comprised: a flipchart of 13 A3 size pages, including text and computer graphics; a verbal presentation of approximately 45 minutes; and a 12 page booklet given to each participant, containing text and black and white diagrams. The flipchart and participant information booklet were designed in consultation with Aboriginal workers, medical and nursing staff, and the sponsor, and were prepared in accordance with international guidelines on the information required for participants in trials of unlicensed vaccines. The materials described the trial vaccine, the diseases that it was designed to prevent, the importance of these diseases in Aboriginal and non-Aboriginal people, the concept of research, the details of participation in the study, potential adverse events, other potential benefits and harms of participation, the voluntary nature of participation, and the need to provide written consent. Particular emphasis was placed on the differentiation between a licensed and unlicensed vaccine, as this concept was considered critical in determining if participants understood the truly experimental nature of participation, and that they would potentially be allowing their children to receive a product of unproven safety and efficacy.

The participant information booklet designed for the nonAboriginal group was based on standard consent materials for vaccine trials developed by the sponsor and comprised text only. Although the format of the materials used for Aboriginal and non-Aboriginal participants was different, the content was the same.

\section{Informed consent procedure}

Before the delivery of the informed consent materials, the rationale for the study and the meaning of research were explained to the Aboriginal participants by an Aboriginal researcher in an open discussion format. The information booklet and flipchart were then explained and discussed in the same informal and interactive setting by an Aboriginal registered nurse. The delivery of the materials took place in groups of between two and nine people, and participants were encouraged to ask questions, offer opinions, and share impressions among themselves and with the study staff. The information booklet was given to participants to take home. Although materials were presented in English to the group of nine participants who spoke English as a second language, extra emphasis was placed on the visual materials and additional time was allowed for discussion and clarification.

For the non-Aboriginal group, the information was delivered in the normal fashion for clinical trials. A registered nurse explained the study to individual participants, answered any questions, and then gave each participant the information booklet to take home.

\section{Evaluation process}

We evaluated the consent materials using a questionnaire and interviews with the Aboriginal participants. The questionnaire was designed in consultation with Aboriginal staff members, medical staff, a literacy professional, and an anthropologist. It consisted of structured and open ended questions $^{8}$ relating to the sociodemographic status of the participants, the design of the informed consent materials, and the major study characteristics. The Aboriginal researcher administered the questionnaire immediately after the delivery of informed consent materials, under supervision of the principal investigator.

Consent materials were delivered to the Aboriginal participants in four groups. Participants in the first three groups were then interviewed on a one-to-one basis. Eight participants consented to their interviews being taped. For the fourth group (nine artists for whom English was their second language), the Aboriginal researcher deemed it inappropriate and discourteous to present the questionnaire formally to each individual in this context. Therefore these women were interviewed together for the first questionnaire and discussed answers among themselves. This group did not consent to being taped.

The same questionnaire was administered to the nonAboriginal group by the principal investigator immediately following the delivery of the consent materials. To determine if the information was retained beyond the initial presentation, participants from both groups were asked the same questions regarding the content of the materials approximately five days later. The non-Aboriginal groups' interviews were not taped.

\section{Ethics approval}

The study was approved by the Central Australian Human Research Ethics Committee and the Central Australian Aboriginal Congress. Written informed consent was obtained from all participants.

\section{Analysis}

Frequency of responses was compared for closed ended questions. Responses to the open ended questions "What is a licensed vaccine?" and "What is an unlicensed vaccine?" were scored independently by two study members for content regarding knowledge of safety, efficacy, and the licensing procedure. A four point scale was used $(l=$ poor understanding, $2=$ some understanding, $3=$ good understanding with some misconceptions, and $4=$ very good understanding with no misconceptions). There was 90\% interobserver agreement. A third study member resolved discrepancies.

Responses to the open ended question relating to potential risks associated with participating in the trial were grouped into the following categories: general vaccine reactions (local and systemic), unexpected vaccine reactions (anaphylaxis and unexpected side effects), blood tests, and potentially painful ear examinations.

Taped interviews were further analysed by a third study member with experience in qualitative research with indigenous people. Each recorded interview was investigated as a communication event using an adapted discourse analysis approach..$^{9-12}$ The following dimensions were considered: the apparent confidence levels of participants, range and use of appropriate terms, intensity of interviewer assistance, internal content coherence (that is, the relation between all the responses/comments of each participant), and the content of both solicited and unsolicited comment. In addition, an unstructured interview was conducted with the Aboriginal interviewer regarding her perceptions of the materials, the evaluation method, and her suggestions for improving approaches to informed consent.

\section{RESULTS}

\section{Questionnaire}

All participants were female and were mothers (table 1). More women in the non-Aboriginal than the Aboriginal group were in paid employment; however more Aboriginal women had undertaken adult training. Only half of the Aboriginal participants spoke English as their first language (table 1). The time taken to deliver the informed consent materials to each group of Aboriginal participants was approximately 60 minutes, whereas 20-25 minutes was spent with each of the non-Aboriginal participants. The median interval between questionnaires 1 and 2 for the Aboriginal and non-Aboriginal participants was 5.5 and 6 days respectively. 
Table 1 Participant details

\begin{tabular}{lll}
\hline & $\begin{array}{l}\text { Aboriginal } \\
\text { participants }(\mathbf{n}=20)\end{array}$ & $\begin{array}{l}\text { Non-Aboriginal } \\
\text { group }(\mathbf{n}=20)\end{array}$ \\
\hline $\begin{array}{l}\text { Female } \\
\text { Age }\end{array}$ & $20(100)$ & $20(100)$ \\
$>16$ years old & $19(95)$ & $20(100)$ \\
$\quad<16$ years old & $1(5)$ & 0 \\
$\begin{array}{l}\text { Employment } \\
\quad \text { Home duties }\end{array}$ & $14(70)$ & $9(45)$ \\
$\quad$ Paid employment & $6(30)$ & $11(55)$ \\
$\begin{array}{l}\text { Education } \\
\text { High school only }\end{array}$ & $1(5)$ & $4(20)$ \\
$\quad \begin{array}{l}\text { Adult training } \\
\text { English as a first }\end{array}$ & $19(95)$ & $16(80)$ \\
language & $10(50)$ & $20(100)$ \\
\hline Numbers in parentheses are percentages. &
\end{tabular}

\section{Understanding}

Most people in both groups recalled ear disease and pneumonia as diseases prevented by the vaccine, however only $55-75 \%$ recalled meningitis, and few recalled sepsis (table 2). Fewer than half of the Aboriginal participants recalled that the vaccine was unlicensed at the initial questionnaire, compared with complete recall for the nonAboriginal group. This was largely due to poor understanding among the artist group: subsequent discussions within this group helped to improve understanding to $100 \%$ at the second questionnaire. Understanding of licensed and unlicensed vaccines improved over time in both groups. However the Aboriginal participants started with a poor understanding, improving to some or good understanding at the second questionnaire. In contrast, the non-Aboriginal group started with a good understanding, improving to good or very good understanding by the second interview (table 2 ).

Recall of the risks of participation was $100 \%$ for general vaccine reactions (local and systemic) in both groups, however recall was poor for unexpected reactions (anaphylaxis and rare events), blood tests, and potentially painful ear examinations in both groups. Recall of the voluntary nature of participation was complete in both groups by the second questionnaire (table 2).

\section{Design of the informed consent materials}

Most participants in the Aboriginal and non-Aboriginal group thought the time taken to deliver the information was appropriate (table 3 ). The flipchart was the preferred medium to deliver the information to the Aboriginal participants, whereas non-Aboriginal participants preferred the complete package of materials. Most participants in each group preferred a doctor to present the information, although the Aboriginal participants preferred an Aboriginal Health Worker to be present as well. More information on reactions and safety issues was requested by $45-50 \%$ of all participants.

\section{Interviews with Aboriginal participants: \\ communication and comprehension}

All taped interviews were with speakers of English as a first language. Most participants had considerable difficulty with the information presented. Half of the participants responded in a tentative and hesitant manner. The majority (6/8) were assisted, particularly regarding the key issue of the distinction between a "licensed" and an "unlicensed" vaccine, which was specifically identified as a point of difficulty and confusion. Comments were made on the number of unknown "big words" that were difficult to recall. There was a clear distinction between the few participants with an apparent familiarity with the vocabulary of the presentation and the majority, who had difficulty articulating their understanding of an unfamiliar, technically complex, discourse on drug research and licensing procedures. Evaluating comprehension using a "yes/no" response did not adequately convey the level of misunderstanding of many participants.

Table 2 Understanding of the content of informed consent materials

\begin{tabular}{|c|c|c|c|c|}
\hline & \multicolumn{2}{|c|}{ Aboriginal participants $(n=20)$} & \multicolumn{2}{|c|}{ Non-Aboriginal participants $(n=20)$} \\
\hline & Questionnaire 1 & Questionnaire 2 & Questionnaire 1 & Questionnaire 2 \\
\hline \multicolumn{5}{|l|}{$\begin{array}{l}\text { Some recall of diseases prevented } \\
\text { by the vaccine }\end{array}$} \\
\hline Ear disease & $20(100)$ & $15(75)$ & $19(95)$ & $19(95)$ \\
\hline Pneumonia & 19 (95) & $18(90)$ & $19(95)$ & $19(95)$ \\
\hline Sepsis & $3(15)$ & $1(5)$ & $4(20)$ & $3(15)$ \\
\hline Meningitis & $15(75)$ & $13(65)$ & $11(55)$ & $12(60)$ \\
\hline $\begin{array}{l}\text { Correctly knew the vaccine was } \\
\text { unlicensed } \\
\text { Understanding of the concept of a } \\
\text { licensed vaccine }\end{array}$ & $9(45)$ & 20 (100) & 20 (100) & $20(100)$ \\
\hline Poor & $10(50)$ & $2(10)$ & $1(5)$ & 0 \\
\hline Some & $6(30)$ & $11(55)$ & $3(15)$ & $3(15)$ \\
\hline Good & $3(15)$ & $6(30)$ & $16(80)$ & $9(45)$ \\
\hline Very good & $1(5)$ & $1(5)$ & 0 & $8(40)$ \\
\hline \multicolumn{5}{|c|}{$\begin{array}{l}\text { Understanding of the concept of an } \\
\text { unlicensed vaccine }\end{array}$} \\
\hline Poor & $12(60)$ & $5(25)$ & 0 & 0 \\
\hline Some & $3(15)$ & $9(45)$ & $10(50)$ & $5(25)$ \\
\hline Good & $5(25)$ & $5(25)$ & $10(50)$ & $14(70)$ \\
\hline Very good & 0 & $1(5)$ & 0 & $1(5)$ \\
\hline \multicolumn{5}{|c|}{$\begin{array}{l}\text { Understanding of potential risks of } \\
\text { participation }\end{array}$} \\
\hline General reactions & $20(100)$ & $20(100)$ & $20(100)$ & $20(100)$ \\
\hline Unexpected reactions & $1(5)$ & $2(10)$ & $7(20)$ & $6(20)$ \\
\hline Blood tests & 0 & 0 & $3(15)$ & 0 \\
\hline Painful ear examination & 0 & 0 & $3(15)$ & $3(15)$ \\
\hline \multicolumn{5}{|c|}{$\begin{array}{l}\text { Knowledge of voluntary nature of } \\
\text { participation }\end{array}$} \\
\hline Yes & $19(95)$ & $20(100)$ & $20(100)$ & $20(100)$ \\
\hline No & $1(5)$ & 0 & 0 & \\
\hline
\end{tabular}




\begin{tabular}{|c|c|c|}
\hline & $\begin{array}{l}\text { Aboriginal } \\
\text { participants }(n=20)\end{array}$ & $\begin{array}{l}\text { Non-Aboriginal } \\
\text { participants }(n=20)\end{array}$ \\
\hline \multicolumn{3}{|l|}{ Length of presentation } \\
\hline Too long & 0 & 0 \\
\hline A bit too long & $5(25)$ & $5(25)$ \\
\hline Just right & $14(70)$ & $15(75)$ \\
\hline A bit too short & $1(5)$ & 0 \\
\hline Too short & 0 & 0 \\
\hline \multicolumn{3}{|l|}{ Most helpful information } \\
\hline Flip chart & $11(55)$ & NA \\
\hline Talking to presenter & $2(10)$ & $5(25)$ \\
\hline Booklet & 0 & $1(5)$ \\
\hline Everything together & $7(35)$ & $14(70)$ \\
\hline \multicolumn{3}{|l|}{ Preferred presenter } \\
\hline Doctor \& AHW & $8(40)$ & 0 \\
\hline Doctor alone & $5(25)$ & $12(60)$ \\
\hline AHW alone & $3(15)$ & 0 \\
\hline Doctor \& nurse & $1(5)$ & 0 \\
\hline Nurse \& AHW & 1 (5) & 0 \\
\hline Doctor \& AHW \& other & $r 1(5)$ & 0 \\
\hline Other & $1(5)$ & $7(35)$ \\
\hline Nurse alone & 0 & $1(5)$ \\
\hline \multicolumn{3}{|l|}{$\begin{array}{l}\text { Further information } \\
\text { requested }\end{array}$} \\
\hline Reactions/safety & $11(55)$ & $9(45)$ \\
\hline Unspecified & $3(15)$ & $1(5)$ \\
\hline None & $6(30)$ & $10(50)$ \\
\hline
\end{tabular}

Several participants indicated a preference for presentations and discussions to be held in groups rather than in oneon-one settings. The majority indicated that they would prefer other family members to be involved in the discussions before they made a decision. Some indicated that they would also seek additional professional opinion.

Suggestions from the participants for improving comprehension of the materials included brevity, minimising text, technical terms and jargon, using visual materials to explain key concepts, presenting information multiple times, using interpreters, presenting to small groups, and using locally meaningful examples. One participant emphasised the importance of the having an Aboriginal Health Worker present together with someone with more authoritative knowledge, such as a doctor or nurse.

\section{Approach used by Aboriginal interviewer}

The Aboriginal interviewer had to be flexible in responding to the requirements of the Aboriginal interviewees. At times, she abandoned the questionnaire method when she judged it to be verging on "unfriendly", and returned to an unstructured discussion. She often assisted those participants who struggled, reassuring them that they were not under scrutiny and that this was not a "test".

\section{DISCUSSION}

These results suggest that a one-off presentation of informed consent materials, even if designed specifically for Aboriginal people, is unlikely to produce the level of "informed consent" that is legally and ethically required under current guidelines for participants in medical research. In comparable indigenous populations it has been argued that morality and ethics are community rather than individual issues. ${ }^{13}$ Our experience supports this view, and also that the current process of promoting individual autonomy over collective or consensual decision making may not reflect the ways in which decisions are made in some cultures. ${ }^{14}$ Rather than relying solely on a single interaction, our results suggest that Aboriginal participants require time, repeated exposure to information, and the opportunity to think about and discuss information with other trusted individuals, before reaching a level of understanding that could be considered satisfactory for informed consent. A staged process should be used beginning with open forums, including structured, planned presentations of the type used in this pilot, to build a broad based understanding of the intended research before approaching individuals.

We were flexible in delivering the consent materials to indigenous participants. Discussions with individuals or small groups were not acceptable to the nine artists who spoke English as a second language. By accommodating their desire to remain as a larger group, we maintained their trust and retained the integrity of their group decision making processes. This component enriched our study, and highlights the heterogeneity that exists within indigenous cultures.

Both groups (Aboriginal and non-Aboriginal) requested more information regarding safety and reactions despite considerable information being provided in the materials. A systematic review of the literature relating to informed consent in non-Aboriginal populations concluded that participants' understanding of the research nature of studies, voluntary participation, the right to withdraw, and available alternative treatments were proportional to the amount of information provided. ${ }^{15}$ However, the provision of too much information was counterproductive. ${ }^{15}$ Although the nonAboriginal participants' understanding was better than that of Aboriginal participants in our study, it remained incomplete, particularly in relation to risks of participation. This group received materials of the same design as those used currently in most industry sponsored clinical trials, suggesting that substantial improvements could be made in conveying information even in settings where language and cultural issues are less substantial.

International guidelines on vaccine research require written information to be provided for participants. However this written material is often lengthy, complex, and inappropriate for many Aboriginal participants. The materials in this study relied on verbal, as well as text and diagrammatic materials. To ensure consistency in the delivery of verbal information, the person delivering the information had to be carefully trained. No Aboriginal participant found the information booklet alone to be sufficient. This is not surprising, given that approximately half of the group spoke English as a second language. Other studies in nonindigenous populations have suggested the use of a readability checklist for written informed consent materials. ${ }^{16} \mathrm{~A}$ similar checklist could be developed for indigenous populations to ensure materials are presented in appropriate English or properly translated. An alternative approach would be to use video or DVD technology, which would ensure a consistent description of research protocols and could potentially be dubbed in appropriate languages.

As this was a pilot study the results are only a guide for the further evaluation of informed consent materials. The participants were not randomly chosen, nor likely to be representative of all Aboriginal people participating in clinical trials. The Aboriginal researcher was a trusted member of the community, which may have improved the ability to convey information. The artist subgroup was given extra verbal information between the first and second interview because the locally resident Aboriginal researcher could not refuse further dialogue during this time. The inability to use the same structured questionnaire for all participants also may have affected the project outcomes. The need to revert to an unstructured discussion for some Aboriginal participants improved the acceptance of the consent process, but made an objective evaluation more difficult. 
Suggestions for delivering informed consent materials for medical research involving indigenous participants

\section{Design}

- Consult widely on the design and content of materials including with: indigenous staff (preferably from the region/s of the participants), health and medical practitioners, language and communications experts.

- Ensure that content abides by international guidelines.

- Identify concepts likely to be unfamiliar and explain them in locally relevant terms.

- Enlist the help of local design experts.

- Use trained interpreters.

- Minimise written text.

- Use multiple means of delivering materials: spoken, written, visual.

- Use clear, appropriate visual materials-for example, videos, diagrams.

- Pre-test the materials.

Delivery

- Have an indigenous health worker and doctor or nurse (preferably known to/trusted by the participants) present the materials.

- Explain the meaning of "research".

- Give initial presentations to community organisations, families, and groups.

- Plan to make presentations more than once.

- Allow time for discussion and questions at presentations.

- Be flexible in regard to presentation setting, time, numbers, etc.

Despite the limits of our investigation, we have formulated suggestions for improving the design and delivery of informed consent materials in indigenous populations (see box). We recommend further research on this important issue.

\section{ACKNOWLEDGEMENTS}

This was a Co-Operative Research Centre for Aboriginal and Tropical Health project, funded by GlaxoSmithKline. We would like to thank the participating Aboriginal organisations, the staff of the maternity and paediatric wards at Alice Springs Hospital for assistance with recruitment, Desley Williams for delivering the materials, Inge Kral for her literacy advice, Yvonne Coleman for administration support and data management, the feasibility study staff, and the participants.

\section{Authors' affiliations}

F M Russell, J R Carapetis, Melbourne University Department of Paediatrics (Centre for International Child Health), Melbourne, Australia J R Carapetis, Murdoch Children's Research Institute, Melbourne, Australia

J R Carapetis, Royal Children's Hospital, Melbourne, Australia H Liddle, T Edwards, J Devitt, Menzies School of Health Research and Cooperative Research Centre for Aboriginal and Tropical Health, Darwin, Australia

T A Ruff, GlaxoSmithKline Biologicals, Melbourne, Australia

\section{REFERENCES}

1 National Health and Medical Research Council. National statement on ethical conduct in research involving humans. Canberra: Commonwealth of Australia, 1999

2 National Health and Medical Research Council. Guidelines on ethical matters in Aboriginal and Torres Strait Islander health research. Canberra: Commonwealth of Australia, 1991.

3 National Health and Medical Research Council. Values and ethics: Guidelines for ethical conduct in Aboriginal and Torres Strait Islander Health Research. Canberra: Commonwealth of Australia, 2003

4 National Health and Medical Research Council. How to present the evidence for consumers: preparation of consumer publications. Canberra: Commonwealth of Australia, 1999.

5 Sless D. Usable written information for patients. Med J Aust 2001; 174:557-8.

6 Buchbinder R, Hall S, Grant G, et al. Readability and content of supplementary written drug information for patients used by Australian rheumatologists. Med J Aust 2001;174:575-8.

7 Paul CL, Redman S, Sanson-Fisher RW. The development of a checklist of content and design characteristics for printed health education materials. Health Promot J Austr 1997:7:153-9.

8 Fallowfield L. Questionnaire design. Arch Dis Child 1995;72:76-9

9 Cooke M. Aboriginal evidence in the cross-cultural courtroom. In: Eades D, ed. Language in evidence. Sydney: UNSW Press, 1995.

10 Eades D. Aboriginal English on trial. In: Eades D, ed. Language in evidence. Sydney: UNSW Press, 1995

11 Eades D. Language in evidence. Sydney: UNSW Press, 1995.

12 Lupton D. Discourse analysis: a new methodology for understanding the ideologies of health and illness. Aust J Public Health 1992;16:145-50.

13 Edwards SJ, Lilford RJ, Thornton J, et al. Informed consent for clinical trials: in search of the "best" method. Soc Sci Med 1998;47:1825-39.

14 Kaufert J, Commanda L, Elias B, et al. Evolving participation of Aboriginal Communities in Health Research Ethics Review: The impact of the Inuvik Workshop. Int J Circumpolar Health 1999;58:134-245.

15 Casteel JK. The ethics of informed consent among storyteller cultures. Int J Circumpolar Health 1998;57(Suppl 1):41-2

16 Tarnowski KJ, Allen DM, Mayhall C, et al. Readability of pediatric biomedical research informed consent forms. Pediatrics 1990;85:58-62. 